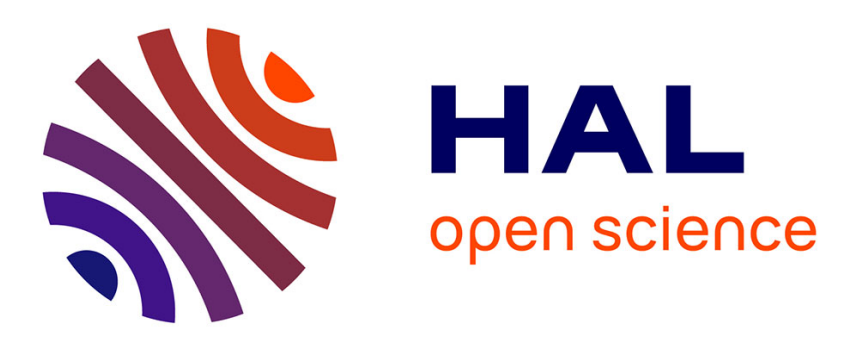

\title{
Optical external efficiency of terahertz quantum cascade laser based on Čerenkov difference frequency generation
}

\author{
A. Hamadou, Jean-Luc Thobel, S. Lamari
}

\section{To cite this version:}

A. Hamadou, Jean-Luc Thobel, S. Lamari. Optical external efficiency of terahertz quantum cascade laser based on Čerenkov difference frequency generation. Journal of Nonlinear Optical Physics and Materials, 2019, 28 (04), pp.1950036. 10.1142/S021886351950036X . hal-02976827

\section{HAL Id: hal-02976827 \\ https://hal.science/hal-02976827}

Submitted on 23 Oct 2020

HAL is a multi-disciplinary open access archive for the deposit and dissemination of scientific research documents, whether they are published or not. The documents may come from teaching and research institutions in France or abroad, or from public or private research centers.
L'archive ouverte pluridisciplinaire HAL, est destinée au dépôt et à la diffusion de documents scientifiques de niveau recherche, publiés ou non, émanant des établissements d'enseignement et de recherche français ou étrangers, des laboratoires publics ou privés. 


\title{
Optical external efficiency of terahertz quantum cascade laser based on Čerenkov difference frequency generation
}

\begin{abstract}
A. Hamadou ${ }^{1,4}$, J.-L. Thobel ${ }^{2}$ and S. Lamari ${ }^{3,4}$
${ }^{1}$ Département de génie civil, Faculté des Sciences et de la Technologie, Université Mohamed Elbachir Elibrahimi, Bordj Bou Arreridj 34000, Algeria

${ }^{2}$ Institut d'Electronique, de Microélectronique et de Nanotechnologie (IEMN), UMR 8520, Université Lille1, Avenue Poincaré, BP 60069, 59652 Villeneuve d’Ascq Cédex, France
\end{abstract}

${ }^{3}$ Département de Physique, Faculté des Sciences, Université Ferhat Abbas Sétif-1, Sétif 19000, Algeria

${ }^{4}$ Laboratoire d'étude des surfaces et interfaces des matériaux solides (LESIMS) Sétif1, Sétif 19000, Algeria

*Corresponding author: abd_hamado@yahoo.fr

Tel: $+213(0) 776937575$ 


\begin{abstract}
In this paper, we consider terahertz sources based on Čerenkov difference frequency generation in a dual-wavelength mid-infrared quantum cascade laser and calculate analytically the optical external efficiency of the out going terahertz wave. The midinfrared pumps operate simultaneously and have a common upper level. For short cavity lengths and low terahertz losses in the substrate, we find that implementation of the Čerenkov emission scheme in terahertz difference frequency generation quantum cascade lasers improves the optical external efficiency dramatically. The maximum value of the latter is close to $19 \%$, but is only attainable if the laser exhibits negligible mid-infrared transmission losses.
\end{abstract}

\title{
Key words:
}

Terahertz quantum cascade laser, difference frequency generation, optical external efficiency, Čerenkov, phased matching. 


\section{Introduction}

The terahertz $(\mathrm{THz})$ region $(0.3-10 \mathrm{THz})$ of the electromagnetic spectrum is extremely important in areas such as spectroscopy, communications, security, and non-destructive testing $^{1-4}$. A variety of $\mathrm{THz}$ sources have already been developed for such applications and most of them are based on the use of quantum cascade (QC) lasers ${ }^{5}$.

Previous designs of THz-QC lasers relied on electron transitions between the quantized energy levels of the conduction band ${ }^{5-8}$. However, these $\mathrm{THz}$ sources suffer from non-radiative relaxation channels and thermal backfilling. Therefore, their performance decreases quite appreciably with increasing temperature. They have on purpose been developed for this wavelength range and so far all of them require cryogenic cooling to operate.

To produce radiation at room temperature in this spectral window a new scheme has recently been used as an alternative. It is based on intra-cavity difference frequency generation (DFG) in dual-wavelength mid-infrared (mid-IR) QC lasers ${ }^{9}$. The key ingredient in the new design is the presence of a giant optical nonlinearity monolithically integrated in the active region. These devices use a dual-stack active region, moreover bound to continuum transitions are designed so as to have a giant intersubband nonlinearity $\chi^{(2)}$ for THz DFG and either two-phonon resonance design or one optical phonon lower states bandwidth ${ }^{10-14}$. This type of design uses just one upper laser state contrary to another design making use of the DFG based on the anticrossed dual-upperstate concept ${ }^{15-16}$. Unfortunately, the main drawbacks of these options are at least twofold: 1) Low power output at room temperature and 2) CW operation. In this regard, the authors in Ref. 17 for instance report a power of just $3 \mu \mathrm{W}$ at $3.6 \mathrm{THz}$. 
The use of the Čerenkov emission is another powerful method enhancing the $\mathrm{THz}$ DFG power output ${ }^{18}$. In this case, $\mathrm{THz}$ radiation is emitted into the substrate and $\mathrm{THz}$ radiation can efficiently be extracted along the entire device length. Čerenkov emission in THz DFG-QC lasers occurs when the phase velocity of the THz wave inside the device substrate is greater than that of the difference frequency nonlinear polarisation wave in the device active region. In this configuration, the phase matching condition is automatically fulfilled under a certain angle to the QC laser pumps path ${ }^{19}$. Čerenkov phase matched $\mathrm{THz}$ wave generation has the advantage of high $\mathrm{THz}$ refractive index and low loss absorption in the substrate.

The schematic illustration of the Čerenkov THz DFG-QC laser is shown in Fig.1. The active region consists of two stacks of stages: In the first one, a double phonon resonance design is in action ${ }^{20}$ and lasing takes place around a wavelength $\lambda_{1} \approx 10.5 \mu \mathrm{m}$ while in the second a bound-to-continuum design is used ${ }^{20}$ and lasing occurs at a wavelength $\lambda_{2}$ about 8.9 $\mu \mathrm{m}$. THz DFG emission occurs into the substrate at Čerenkov angle $\theta_{C}$. The propagation angle of the Čerenkov $\mathrm{THz}$ emission with respect to the QC laser cavity is defined as $\theta_{C}=\cos ^{-1}\left(n_{g} / n_{\text {sub }}\right)$, where $n_{\mathrm{g}}$ and $n_{\text {sub }}$ are respectively the group velocity index of the mid-IR pumps and the $\mathrm{THz}$ refractive index of the substrate. For more technical details on the structure, we kindly refer the reader to the published literature ${ }^{18}$, $21-23$.

In THz DFG-QC lasers, the mid-IR-to-THz conversion (defined as the ratio of the THz power output to the product of the two powers of the mid-IR pumps) and the wallplug (defined as the ratio of the optical output power to the input electrical power) efficiencies play an important role that partly determine the device's performance and 
have already been investigated experimentally in the case of a Čerenkov THz DFG-QC

laser $^{23-24}$. At room temperature, the maximum mid-IR-to-THz conversion efficiencies exceed $0.8 \mathrm{~mW} / \mathrm{W}^{2}$ and $0.35 \mathrm{~mW} / \mathrm{W}^{2}$ for pulsed ${ }^{24}$ and $\mathrm{CW}$ operations ${ }^{22}$ respectively, while the best wall-plug efficiencies of mid-IR QC lasers exceed $0.7 \times 10^{-3} \%$ and $0.8 \times 10^{-4} \%$ for pulsed $^{17}$ and CW operations ${ }^{22}$ respectively.

Equally important for the DFG-QC laser operation is the optical external efficiency, known as optical extraction efficiency in the laser physics literature ${ }^{25}$. This parameter is useful for designing THz DFG-QC lasers and optimising their performance. In this paper, we calculate analytically the optical external efficiency for the THz-QC laser based on intra-cavity Čerenkov DFG, using a rate equation model.

Our paper is organized as follows: Section 2 describes the rate equations model of the two mid-IR mode pumps used to derive our results. In addition, this section also contains the steady state solutions above the second QC laser threshold. Section 3 contains the analytical derivation of the optical external efficiency for the THz-QC laser-based on Čerenkov DFG. Finally, Section 4 concludes our paper and highlights our main findings.

\section{The model}

The simplified energy level scheme of one period of the bound-to-continuum active region given in Fig. 2 is used to set up the rate equations. The upper and lower states for wavelength pump $\lambda_{1}=10.5 \mu \mathrm{m}$ will be taken as levels 4 and 3 respectively, while 4 and 2 will be for wavelength pump $\lambda_{2}=8.9 \mu \mathrm{m}$. For mode 1 of wavelength $10.5 \mu \mathrm{m}$, the first excited state denoted 2 and the ground state denoted 1 are used to empty the lower state through emission of longitudinal optical (LO) phonons. The same ground state, through 
LO-phonon emission, is also used to empty the lower state of the lasing mode 2 at wavelength $8.9 \mu \mathrm{m}$. The $\mathrm{THz}$ radiation is generated via the DFG process between levels 3 and 2.

Fig. 2 also presents a sketch of our structure and displays in red and blue the radiative transitions and in grey the non-radiative ones due to phonon scattering. At this stage note that intersubband phonon scattering occurs not only between levels 4 and 1, and 4 and 2 on one hand but also between 4 and 3 as well. In this regard, we also point out the importance of phonon scattering which has been shown to be the main competing nonradiative process in mid-IR QC lasers. We restrict the rate equations model to a threelevel system. The dynamic variables comprise the respective instantaneous numbers of electrons in each level denoted by $N_{2}, N_{3}$, and $N_{4}$ and the photon numbers $S^{(1)}$ and $S^{(2)}$ for the two mid-IR pumps. In the following, we assume a unity injection efficiency for the current into the upper laser level, ignore emission of radiation in the double phonon resonance active region and neglect the thermal backfilling transition times.

The system may be described by the five following rate equations ${ }^{26-28}$ :

$$
\begin{aligned}
& \frac{d N_{4}}{d t}=\frac{I}{e}-\frac{N_{4}}{\tau_{4}}-G^{(1)}\left(N_{4}-N_{3}\right) S^{(1)}-G^{(2)}\left(N_{4}-N_{2}\right) S^{(2)} \\
& \frac{d N_{3}}{d t}=\frac{N_{4}}{\tau_{43}}-\frac{N_{3}}{\tau_{3}}+G^{(1)}\left(N_{4}-N_{3}\right) S^{(1)}, \\
& \frac{d N_{2}}{d t}=\frac{N_{4}}{\tau_{42}}+\frac{N_{3}}{\tau_{32}}-\frac{N_{2}}{\tau_{21}}+G^{(2)}\left(N_{4}-N_{2}\right) S^{(2)}, \\
& \frac{d S^{(1)}}{d t}=N_{p} G^{(1)}\left(N_{4}-N_{3}\right) S^{(1)}-\frac{S^{(1)}}{\tau_{p}^{(1)}}+N_{p} \beta^{(1)} \frac{N_{4}}{\tau_{s p}^{(1)}}
\end{aligned}
$$




$$
\frac{d S^{(2)}}{d t}=N_{p} G^{(2)}\left(N_{4}-N_{2}\right) S^{(2)}-\frac{S^{(2)}}{\tau_{p}^{(2)}}+N_{p} \beta^{(2)} \frac{N_{4}}{\tau_{s p}^{(2)}}
$$

Where $I$ denotes the electron current being pumped into the upper level, $e$ is the absolute value of the electronic charge. In addition, in the above equations we introduced the gain coefficient per stage $G^{(i)}=\Gamma^{(i)} c^{\prime} \sigma^{(i)} / V$ where $\Gamma^{(i)}$ is the mode confinement factor for wavelength $\lambda_{i}(i=1,2), c^{\prime}$ is the average velocity of light in the system, $\sigma^{(i)}$ the stimulated emission cross section for the transition corresponding to the wavelength $\lambda_{i}$ and $V=N_{p} W L L_{p}$ represents the whole volume $V$ of the active region where $W$ and $L$ stand for the lateral dimensions of the cavity while $N_{p}$ and $L_{p}$ are the number of stages and length of each one of these. The important parameter $\beta^{(i)}$ in Eqs.(1d) and (1e) is the coupling efficiency of spontaneous emission in $\operatorname{mode}^{29}(i)$, i.e. the proportion, among all spontaneously emitted photons, of those which are emitted in the cavity mode labeled $(i)$.

The system dynamics is mainly determined by the six non radiative scattering times denoted by $\tau_{43}, \tau_{42}, \tau_{41}, \tau_{32}, \tau_{31}$, and $\tau_{21}$ that are due to LO-phonon emission and the radiative spontaneous relaxation times $\tau_{s p}^{(i)}$ for the two transitions involved.

To complete the picture, we take into consideration for both mid-IR pumps the finite lifetimes of the photons denoted by $\tau_{p}^{(i)}$ and given as functions of the waveguide losses $\alpha_{w}^{(i)}$ and the mirrors losses by 


$$
\tau_{p}^{(i)}=\frac{2 L}{c^{\prime}} \frac{1}{\left(2 L \alpha_{w}^{(i)}-\ln \left(R_{1}^{(i)}\right)\right)} \cdot \quad(i=1,2)
$$

For the sake of convenience, let us also introduce the lifetimes $\tau_{4}$ and $\tau_{3}$ of the levels 4 and 3 which we write as $\tau_{4}=1 /\left(1 / \tau_{43}+1 / \tau_{42}+1 / \tau_{41}\right)$ and $\tau_{3}=1 /\left(1 / \tau_{32}+1 / \tau_{31}\right)$.

In the steady state, all the time derivatives in the rate equations given above vanish. An analytical solution, above the second QC laser threshold, of the system becomes then feasible. The following expressions for the intersubband population inversions $\Delta N^{(1)}$ and $\Delta N^{(2)}$, and the nontrivial stable solutions for the photon numbers $S^{(1)}$ and $S^{(2)}$ of the two mid-IR pumps ensue:

$$
\begin{aligned}
& \Delta N^{(1)}=\frac{I_{t h}^{(1)}}{e} \tau_{4}\left(1-\frac{\tau_{3}}{\tau_{43}}\right), \\
& \Delta N^{(2)}=\frac{I_{t h}^{(2)}}{e} \tau_{4}\left(1-\frac{\tau_{3} \tau_{21}}{\tau_{43} \tau_{32}}-\frac{\tau_{21}}{\tau_{42}}\right), \\
& S^{(1)}=S_{s a t}^{(1)} \frac{-a_{1}+\left(a_{1}^{2}-4 a_{0}\right)^{1 / 2}}{2}, \\
& S^{(2)}=S_{s a t}^{(2)} \frac{\frac{I}{I_{t h}^{(2)}}-1+\left(\frac{I}{I_{t h}^{(2)}} \delta_{2}-1\right) \frac{S^{(1)}}{S_{s a t}^{(1)}}}{1+\delta_{0} \frac{S^{(1)}}{S_{s a t}^{(1)}}}
\end{aligned}
$$

where the threshold current $I_{t h}^{(1)}$ and $I_{t h}^{(2)}$ are expressed as ${ }^{26}$ 


$$
\begin{aligned}
& I_{t h}^{(1)}=\frac{e}{N_{p} G^{(1)} \tau_{p}^{(1)} \tau_{4}\left(1-\frac{\tau_{3}}{\tau_{43}}\right)}, \\
& I_{t h}^{(2)}=\frac{e}{N_{p} G^{(2)} \tau_{p}^{(2)} \tau_{4}\left(1-\frac{\tau_{3} \tau_{21}}{\tau_{43} \tau_{32}}-\frac{\tau_{21}}{\tau_{42}}\right)},
\end{aligned}
$$

and the photon saturation numbers for the two mid-IR pumps are given by

$$
\begin{aligned}
& S_{\text {sat }}^{(1)}=\frac{1}{\tau_{4}\left(1+\frac{\tau_{3}}{\tau_{42}}+\frac{\tau_{3}}{\tau_{41}}\right) G^{(1)}}, \\
& S_{\text {sat }}^{(2)}=\frac{1}{\tau_{4}\left(1+\frac{\tau_{3} \tau_{21}}{\tau_{43} \tau_{31}}+\frac{\tau_{21}}{\tau_{41}}\right) G^{(2)}} .
\end{aligned}
$$

In Eq.(4a), the parameters $a_{0}$ and $a_{1}$ are given by the relations

$$
\begin{aligned}
& a_{0}=-\frac{\left(1-\delta_{1}\right)}{\delta_{0} \delta_{2}} \frac{I_{t h}^{(2)}}{I_{t h}^{(1)}}+\frac{1}{\delta_{0} \delta_{2}}-\frac{I}{I_{t h}^{(1)}} \frac{\delta_{1}}{\delta_{0} \delta_{2}}, \\
& a_{1}=\left(\frac{\delta_{1}}{\delta_{0} \delta_{2}}-\frac{1}{\delta_{2}}\right) \frac{I_{t h}^{(2)}}{I_{t h}^{(1)}}+\frac{1}{\delta_{2}}+\frac{1}{\delta_{0}}-\frac{I}{I_{t h}^{(1)}} \frac{\delta_{1}}{\delta_{0}} .
\end{aligned}
$$

where the parameters $\delta_{0}, \delta_{1}$ and $\delta_{2}$ are defined as

$$
\delta_{0}=\frac{\frac{\tau_{3}}{\tau_{4}}\left(1+\frac{\tau_{21}}{\tau_{31}}+\frac{\tau_{21}}{\tau_{41}}\right)}{\left(1+\frac{\tau_{3}}{\tau_{42}}+\frac{\tau_{3}}{\tau_{41}}\right)\left(1+\frac{\tau_{3} \tau_{21}}{\tau_{43} \tau_{31}}+\frac{\tau_{21}}{\tau_{41}}\right)},
$$




$$
\begin{aligned}
& \delta_{1}=\frac{1}{\left(\frac{\tau_{4}}{\tau_{21}}+\frac{\tau_{4} \tau_{3}}{\tau_{43} \tau_{31}}+\frac{\tau_{4}}{\tau_{41}}\right)}, \\
& \delta_{2}=\frac{\tau_{3}\left(1-\frac{\tau_{21}}{\tau_{32}}-\frac{\tau_{21}}{\tau_{42}}\right)}{\tau_{4}\left(1-\frac{\tau_{3} \tau_{21}}{\tau_{43} \tau_{32}}-\frac{\tau_{21}}{\tau_{42}}\right)\left(1+\frac{\tau_{3}}{\tau_{42}}+\frac{\tau_{3}}{\tau_{41}}\right)} .
\end{aligned}
$$

Note that the second QC laser threshold is defined by ${ }^{26}$

$$
\tilde{I}_{t h}^{(2)}=\left(1+\frac{1}{\delta_{2}}\left(\frac{I_{t h}^{(2)}}{I_{t h}^{(1)}}-1\right)\right) I_{t h}^{(1)} .
$$

Let us stress that $\tilde{I}_{t h}^{(2)}$ is the actual physical threshold current and is to be distinguished from the threshold $I_{t h}^{(2)}$, introduced for convenience, and derived in the absence of any optical field in the cavity. Let us also mention that above the second QC laser threshold both modes are present, the two mid-IR pumps therefore oscillate simultaneously.

Our computation uses the following QC laser parameters at room temperature as reported in $\operatorname{Refs}{ }^{18,30}: \tau_{4}=1 \mathrm{ps}, \tau_{43}=12.5 \mathrm{ps}, \tau_{42}=2.3 \mathrm{ps}, \tau_{41}=2.1 \mathrm{ps}, \tau_{3}=0.13 \mathrm{ps}, \tau_{32}=3$ ps, $\quad \tau_{31}=0.15 \quad$ ps, $\quad \tau_{21}=0.18 \quad$ ps, $\quad \tau_{p}^{(1)} \approx \tau_{p}^{(1)} \approx 7.7 \quad$ ps, $\quad \alpha_{w}^{(1)} \approx \alpha_{w}^{(2)}=8 \quad \mathrm{~cm}^{-1}$, $R_{1}^{(1)}=R_{1}^{(2)}=R_{1}=0.29, L=2 \mathrm{~mm}, W=24 \mu \mathrm{m}$. The physical threshold current densities at wavelengths $\lambda_{1}=10.5 \mu \mathrm{m}$ and $\lambda_{2}=8.9 \mu \mathrm{m}$ are $J_{t h}^{(1)} \approx 6 \mathrm{kA} / \mathrm{cm}^{2}$ and $\tilde{J}_{t h}^{(2)} \approx 9.3 \mathrm{kA} / \mathrm{cm}^{2}$ respectively.

\section{Derivation of the optical external efficiency for the Čerenkov THz DFG-QC laser}


The system of Eqs.(1) describes the amplification of the mid-IR radiation fields inside the nonlinear active medium and will now be used to derive the Čerenkov power coming through the front facet of the substrate $P_{o u t}^{(T H z)}$ and the optical external efficiency $\eta_{e x t}^{(T H z)}$.

DFG is a nonlinear optical process in which two beams at angular frequencies $\omega_{1}$ and $\omega_{2}$, in our case both in the mid-IR range, interact in a nonlinear medium having an effective second-order nonlinear susceptibility $\chi^{(2)}$ to produce radiation at a $\mathrm{THz}$ frequency $\omega_{T H z}=\omega_{1}-\omega_{2}$. The wave-vector mismatch of this process is $\Delta k=k_{1}-k_{2}-k_{T H z}$, where $k_{i}$, is the wave vector of the mid-IR pump mode at frequency $\omega_{i}$ and $k_{T H z}$ is the wave vector at $\mathrm{THz}$ frequency ${ }^{31}$. THz DFG radiation occurs in the nonlinear medium, along the whole waveguide length in these devices.

It is well established that the efficiency of the DFG process critically depends on the matching of phase velocities of interacting waves ${ }^{31}$. In order to produce perfect phase matching $\Delta k=0$, we use in this work the Čerenkov DFG scheme as mentioned in section 1. We use here a semi-insulating $(\mathrm{SI}) \mathrm{InP}$ substrate and the generated $\mathrm{THz}$ radiation is emitted at the Čerenkov angle $\theta_{\mathrm{C}}$. For example for the refractive index in the nonlinear active region $\mathrm{n}_{\mathrm{g}}=3.37$ and at a wavelength of $60 \mu \mathrm{m}$, SI- InP substrate has a refractive index of about $\mathrm{n}_{\text {sub }} \sim 3.6$ which results in a $21^{\circ}$ Čerenkov angle in the substrate ${ }^{18}$.

In most cases, absorption degrades the performance of devices operating at $\mathrm{THz}$ frequencies and our aim here is to estimate by how much. To quantify the degradation, we derive the optical external efficiency while keeping the loss terms in the nonlinear active region and the substrate. 
The output power of the THz wave produced through Čerenkov DFG is obtained from the well-known formula ${ }^{31-32}$, including the effects of absorption in the non depleted pump approximation and assuming perfect phase matching $\Delta k=0$ :

$$
P_{\text {out }}^{(T H z)} \propto\left|\chi^{(2)}\right|^{2} P^{(1)} P^{(2)} \frac{1+\mathrm{e}^{\left(-\alpha_{t o t} L\right)}-2 \mathrm{e}^{\left(-\alpha_{t o t} L / 2\right)}}{(\Delta \alpha L / 2)^{2}}\left(1-R_{\text {sub }}^{(T H z)}\right) \int_{0}^{L} e^{-\alpha_{s u b}^{(T H z)} z \cos \theta_{c}} d z .
$$

Here, $P^{(i)}(i=1,2)$ is the mid-IR pump beam power at $\omega_{i}$ and can be deduced numerically from Eqs. (4) by using the relationship between power in units of $\mathrm{W}$ and photon number in the cavity $P^{(i)}=c^{\prime} \hbar \omega_{i} S^{(i)} / L^{33}$. Here, $\hbar \omega_{i}$ is the energy of the emitted photon at frequency $\omega_{i}$. In Eq.(10), we denoted by $\alpha_{t o t}$ the $\operatorname{sum} \alpha_{t o t}=\alpha_{w}^{(1)}+\alpha_{w}^{(2)}$ where $\alpha_{w}^{(i)}(i=1,2)$ is the loss due to absorption in the nonlinear waveguide at $\omega_{i}$. Moreover in the integrand, $\alpha_{\text {sub }}^{(T H z)}$ stands for the loss due to absorption in the substrate, and $\theta_{c}$ is the Čerenkov angle, while in the prefactor $R_{s u b}^{(T H z)}$ is the output coupling at THz frequency in the substrate.

The intersubband nonlinear susceptibility $\chi^{(2)}$ for the THz DFG is given in the case of a bound-to-continuum active region as ${ }^{10}$ :

$$
\chi^{(2)}=\frac{e^{3}}{\varepsilon_{0} \hbar^{2}} \frac{z_{43} z_{32} z_{24}}{\left(\omega_{T H z}-\omega_{32}+i \Gamma_{32}\right)}\left(\frac{\Delta N^{(1)}}{-\omega_{1}+\omega_{43}+i \Gamma_{34}}+\frac{\Delta N^{(2)}}{\omega_{2}-\omega_{42}+i \Gamma_{24}}\right),
$$

where $e z_{i j}$ is the dipole matrix element, $\omega_{i j}$ and $\Gamma_{i j}$ are respectively the frequency and broadening of the transition between states $i$ and $j$, while $\varepsilon_{0}$ and $\hbar$ have their usual meaning. 
The maximum of the Čerenkov power $P_{\max }^{(T H z)}$ in the substrate is attained when all the waveguide and substrate losses are zero (i.e., $\alpha_{w}^{(1)}=\alpha_{w}^{(2)}=\alpha_{s u b}^{(T H z)}=0$ ), and the output coupling coefficients $R_{1}^{(1)}$ and $R_{1}^{(2)}$ for both mid-IR modes pumps approach $100 \%$ (i.e., $\left.R_{1}^{(1)}=R_{1}^{(2)}=R \rightarrow 1\right)$. In these conditions the intersubband population inversions for both modes become $\Delta N_{U L}^{(1)}=\left(-W L_{p} \ln (R)\right) /\left(\Gamma \sigma^{(1)}\right)$ and $\Delta N_{U L}^{(2)}=\left(-W L_{p} \ln (R)\right) /\left(\Gamma \sigma^{(2)}\right)$, and the mid-IR pumps powers $P^{(i)}$ represent the powers $P_{U L}^{(i)}$ transferred into the upper laser level, ie., the maximal mid-IR powers in the cavity. Thus, the maximum Čerenkov power $P_{\max }^{(T H z)}$ is obtained from Eq. (10) by making $\chi_{U L}^{(2)}$ and $P_{U L}^{(i)}$ replace $\chi^{(2)}$ and $P^{(i)}$ respectively

$$
P_{\max }^{(T H z)} \propto L\left|\chi_{U L}^{(2)}\right|^{2} P_{U L}^{(1)} P_{U L}^{(2)},
$$

where $\chi_{U L}^{(2)}$ is the nonlinear susceptibility in the absence of losses and is given by

$$
\chi_{U L}^{(2)}=\frac{e^{3}}{\varepsilon_{0} \hbar^{2}} \frac{z_{43} z_{32} z_{24}}{\left(\omega_{T H z}-\omega_{32}+i \Gamma_{32}\right)}\left(\frac{\Delta N_{U L}^{(1)}}{-\omega_{1}+\omega_{43}+i \Gamma_{34}}+\frac{\Delta N_{U L}^{(2)}}{\omega_{2}-\omega_{42}+i \Gamma_{24}}\right) .
$$

We now derive a general expression for the optical external efficiency of Čerenkov THz DFG-QC laser $\eta_{e x t}^{(T H z)}$ which is an important parameter characterizing the optical performance of a laser. This quantity is defined as the ratio of the THz DFG-QC laser output power and the maximum $\mathrm{THz}$ DFG-QC laser power in the substrate i.e. $\eta_{e x t}^{(T H z)}=P_{o u t}^{(T H z)} / P_{\max }^{(T H z)}$. Using Eqs. (10) and (12), we get 
$\eta_{\text {ext }}^{(T H z)}=\left|\frac{\chi^{(2)}}{\chi_{U L}^{(2)}}\right|^{2} \frac{P^{(1)} P^{(2)}}{P_{U L}^{(1)} P_{U L}^{(2)}} \frac{1+\mathrm{e}^{\left(-\alpha_{t o t} L\right)}-2 \mathrm{e}^{\left(-\alpha_{t o t} L / 2\right)}}{\left(\alpha_{t o t} L / 2\right)^{2}}\left(1-R_{s u b}^{(T H z)}\right) \frac{1}{L} \int_{0}^{L} e^{-\alpha_{s u b}^{(T H z)} z \cos \theta_{c}} d z$.

In the case where the two mid-IR pumps frequencies in the waveguide laser are in resonance with the intersubband transitions, ie., $\omega_{1} \approx \omega_{43}, \omega_{2} \approx \omega_{42}$ and for $\Gamma_{43} \approx \Gamma_{42}$, the ratio $\chi^{(2)} / \chi_{U L}^{(2)}$ simplifies to

$$
\frac{\chi^{(2)}}{\chi_{U L}^{(2)}}=\frac{\Delta N^{(1)}+\Delta N^{(2)}}{\Delta N_{U L}^{(1)}+\Delta N_{U L}^{(2)}}
$$

Multiplying and dividing Eq.(14) by $(1-R)^{2}$ and using the approximation $(1-R) \approx|\ln (R)|$, after some algebra, we get for $\eta_{\text {ext }}^{(T H z)}$ the following expression:

$$
\eta_{e x t}^{(T H z)}=\left(\frac{2 L}{c^{\prime} \tau_{p}^{(1)}} \frac{\left(1-\frac{\tau_{3}}{\tau_{43}}\right)+\frac{I_{t h}^{(2)}}{I_{t h}^{(1)}}\left(1-\frac{\tau_{3} \tau_{21}}{\tau_{43} \tau_{32}}-\frac{\tau_{21}}{\tau_{42}}\right)}{\left(1-\frac{\tau_{3}}{\tau_{43}}\right)+\frac{I_{t h}^{(2)}}{I_{t h}^{(1)}} \frac{\tau_{p}^{(2)}}{\tau_{p}^{(1)}}\left(1-\frac{\tau_{3} \tau_{21}}{\tau_{43} \tau_{32}}-\frac{\tau_{21}}{\tau_{42}}\right)}\right)^{2} \eta_{\mathrm{int}}^{(1)} \eta_{\mathrm{int}}^{(2)} \ell\left(1-R_{\text {sub }}^{(T H z)}\right)
$$

In the above expression, $\ell$ serves as the relative efficiency function in the presence of losses and can be expressed as 


$$
\ell=\frac{1+\mathrm{e}^{\left(-\alpha_{t o t} L\right)}-2 \mathrm{e}^{\left(-\alpha_{t o t} L / 2\right)}}{\left(\alpha_{t o t} L / 2\right)^{2}} \frac{\left(1-e^{-\alpha_{s u b}^{(T H z)} L \cos \theta_{c}}\right)}{\alpha_{s u b}^{(T H z)} L \cos \theta_{c}} .
$$

In the absence of any optical losses, the relative efficiency approaches unity.

In Eq.(16), the factors $\eta_{\text {int }}^{(1)}$ and $\eta_{\text {int }}^{(2)}$ are the mid-IR optical internal efficiencies at wavelengths $\lambda_{1}$ and $\lambda_{2}$ and are given by:

$$
\begin{aligned}
& \eta_{\mathrm{int}}^{(1)}=\frac{c^{\prime} \tau_{p}^{(1)} \delta_{0}}{2 L \delta_{1}} \frac{S^{(1)}}{S_{s a t}^{(1)}} \frac{I_{t h}^{(1)}}{I}, \\
& \eta_{\mathrm{int}}^{(2)}=\frac{c^{\prime} \tau_{p}^{(2)} \delta_{0}}{2 L \delta_{2}} \frac{S^{(2)}}{S_{s a t}^{(2)}} \frac{I_{t h}^{(2)}}{I} .
\end{aligned}
$$

In the case where $\alpha_{w}^{(1)} \approx \alpha_{w}^{(2)}$, the photon lifetimes for both modes are identical, i.e., $\tau_{p}^{(1)} \approx \tau_{p}^{(2)}$, and the optical external efficiency is well approximated by

$$
\eta_{e x t}^{(T H z)} \approx \eta_{e x t, \max }^{(T H z)} \ell
$$

where

$$
\eta_{\text {ext, } \max }^{(T H z)} \approx \frac{\delta_{0}^{2}}{\delta_{1} \delta_{2}} \frac{S^{(1)}}{S_{s a t}^{(1)}} \frac{S^{(2)}}{S_{s a t}^{(2)}} \frac{I_{t h}^{(1)} I_{t h}^{(2)}}{I^{2}}\left(1-R_{s u b}^{(T H z)}\right)
$$

In the above equation, $\eta_{e x t \text { max }}^{(T H z)}$ denotes the $\mathrm{THz}$ optical external efficiency when all the losses vanish, so that it represents the best performance. 
As we can see from Eq. (16) the optical external efficiency for Čerenkov $\mathrm{THz}$ DFG depends on the materials parameters of the QC laser and can be controlled either through the cavity length or by the current injection.

We show in Fig.3 the variation of the relative efficiency as a function of the $\mathrm{THz}$ losses in the substrate with and without mid-IR losses. In the absence of any losses, the relative efficiency approaches unity. It decreases when losses in the substrate increase. The mid-IR losses reduce dramatically the relative efficiency and hence the laser performance.

In Fig.4, the THz-optical external efficiency is plotted as a function of current injection for different cavity lengths at three values of the $\mathrm{THz}$ losses in the substrate. As shown on the different panels, at the second QC laser threshold, the THz-optical external efficiency is zero and it increases when the current injection is increased. For lower values of the cavity length, the photon lifetime in the cavity decreases, thus reducing the threshold current and therefore directly enhancing the THz-optical external efficiency which is very sensitive to the $\mathrm{THz}$ losses in the substrate. For example, for $L=1 \mathrm{~mm}$ and at $I=12 \mathrm{~A}$, the THz-optical external efficiency increases by a factor of $\sim 1.4$ times for $\alpha=10 \mathrm{~cm}^{-1}$ and 1.7 times for $\alpha=5 \mathrm{~cm}^{-1}$ compared to $\alpha=20 \mathrm{~cm}^{-1}$.

Fig.5 depicts contour lines of THz-optical external efficiency $\eta_{e x t}^{(T H z)}$ as a function of the cavity length and $\mathrm{THz}$ loss in the substrate. We observe that the impact of the higher $\mathrm{THz}$ loss in the substrate on the THz-optical external efficiency can be compensated by 
decreasing the cavity length. The optimal choice of $L<2 \mathrm{~mm}$ and $\alpha_{\text {sub }}\left\langle 5 \mathrm{~cm}^{-1}\right.$ results in the greatest value of $\left.\eta_{e x t}^{(T H z)}\right\rangle 5 \%$. It becomes lower than $4 \%$ at $\alpha_{\text {sub }}>10 \mathrm{~cm}^{-1}$ for a cavity larger than $2 \mathrm{~mm}$.

Fig.6 shows the mid-IR reflecting power $R_{1}$ dependence of the optical external efficiencies of the mid-IR pumps powers $\left(\eta_{\text {ext }}^{(i)}=\eta_{\text {int }}^{(i)}\left(1-R_{1}\right)\right.$ and THz DFG-QC laser. We find that a maximum $\mathrm{THz}$ optical external efficiency of $19 \%$ is only attainable if the laser exhibits no mid-IR transmission loss $\left(R_{1}=1\right)$. In this situation the optical external efficiencies for both mid-IR modes pumps approach zero and the maxima of the mid-IR powers are located in the nonlinear active region.

We show in Fig.7 the variation of the THz-optical external efficiency as a function of the $\mathrm{THz}$ losses in the substrate for two mid-IR reflecting power $R_{1}$. It is worthwhile to stress the strong decrease of $\eta_{e x t}^{(T H z)}$ as the THz losses in the substrate increase from its minimal value 0 upward. We also see that $\eta_{e x t}^{(T H z)}$ depends on the $R_{1}$ value. By increasing the $\mathrm{THz}$ losses from 0 to $40 \mathrm{~cm}^{-1}$, the THz-optical external efficiency decreases from $19 \%$ to $5 \%$ for $R_{1}=0.29$ and from $24 \%$ to $6.5 \%$ for $R_{1}=1$. This decrease is due to the strong dependence of the $\eta_{e x t}^{(T H z)}$ on the relative efficiency $\ell$ which is strongly affected by the value of the THz loss in substrate, as shown in Fig.3. 


\section{Conclusion}

In summary, we have studied the performance of the Terahertz sources based on Čerenkov difference frequency generation in dual-wavelengths mid-IR QC lasers. We have developed an analytical scheme leading to a closed form determination of the THzoptical external efficiency. With the present design and physical parameters, the injection current and the cavity length are found to play a central role on the performance of the Čerenkov THz QC laser. We also find that the drawbacks on the THz-optical external efficiency due to important $\mathrm{THz}$ losses in the substrate can be alleviated by decreasing the cavity length.

\section{Acknowledgment}

The authors are much indebted to Dr. F. Dessenne from IEMN, University Lille 1, for his generous help. Moreover, S. Lamari and A. Hamadou are grateful to the Algerian Ministry of Higher Education for a research grant, CNEPRU B00L02UN190120150003. 


\section{References}

1. B. Ferguson and X.-C. Zhang, Materials for terahertz science and technology, Nat. Mater. 1 (2002) 26.

2. M. Tonouchi, Cutting-edge terahertz technology, Nat. Photon. 1 (2007) 97.

3. P.U. Jepsen, D.G. Cooke and M. Koch, Terahertz spectroscopy and imagingModern techniques and applications, Laser Photon. Rev. 5 (2011) 124.

4. P. Dean, A. Valavanis, J. Keeley, K. Bertling, Y.L. Lim, R. Alhathlool, A.D. Burnett, L.H. Li, S.P. Khanna, D. Indjin, T. Taimre, A.D. Rakić, E.H. Linfield and A.G. Davies, Terahertz imaging using quantum cascade lasers- a review of systems and applications, J. Phys. D: Appl. Phys. 47 (2014) 374008.

5. R. Köhler, A. Tredicucci, F. Beltram, H.E. Beere, E.H. Linfield, A.G. Davies, D.A. Ritchie, R.C. Iotti and F. Rossi, Terahertz semiconductor-heterostructure laser, Nature. 417 (2002) 156.

6. B.S. Williams, H. Callebaut, S. Kumar, Q. Hu and J.L. Reno, 3.4-THz quantum cascade laser based on longitudinal-optical-phonon scattering for depopulation, Appl. Phys. Lett. 82 (7) (2003) 1015.

7. S. Fathololoumi, E. Dupont, C.W.I. Chan, Z.R. Wasilewski, S.R. Laframboise, D. Ban, A. Mátyás, C. Jirauschek, Q. Hu and H.C. Liu, Terahertz quantum cascade lasers operating up to $\sim 200 \mathrm{~K}$ with optimized oscillator strength and improved injection tunneling, Opt. Express. 20 (2012)3866.

8. M.S. Vitiello, G. Scalari, B. Williams and P. De Natale, Quantum cascade lasers: 20 years of challenges, Opt. Express. 23 (2015) 5167.

9. M.A. Belkin, F. Capasso, A. Belyanin, D.L. Sivco, A.Y. Cho, D.C. Oakley, C.J. Vineis and G.W. Turner, Terahertz quantum-cascade laser source based on intracavity difference-frequency generation, Nat. Photon. 1 (2007) 288.

10. M.A. Belkin, F. Capasso, F. Xie, A. Belyanin, M. Fischer, A. Wittmann and J. Faist, Room temperature terahertz quantum cascade laser source based on intracavity difference-frequency generation, Appl. Phys. Lett. 92 (2008) 201101.

11. Y. Jiang, K. Vijayraghavan, S. Jung, F. Demmerle, G. Boehm, M.C. Amann and M.A. Belkin, External cavity terahertz quantum cascade laser sources based on intra-cavity frequency mixing with $1.2-5.9 \mathrm{THz}$ tuning range, J. Opt. 16 (2014) 094002. 
12. S. Jung, A. Jiang, Y. Jiang, K. Vijayraghavan, X. Wang, M. Troccoli and M.A. Belkin, Broadly tunable monolithic room temperature terahertz quantum cascade laser sources, Nat. Commun. 5 (2014) 4267.

13. Q.Y. Lu, S. Slivken, N. Bandyopadhyay, Y. Bai and M. Razeghi, Widely tunable room temperature semiconductor terahertz source, Appl. Phys. Lett. 105 (2014) 201102.

14. M.A. Belkin and F. Capasso, New frontiers in quantum cascade lasers: high performance room temperature terahertz sources, Phys. Sci. 90 (2015) 118002.

15. K. Fujita, T. Edamura, S. Furuta and M. Yamanishi, High performance, homogeneous broad-gain quantum cascade lasers based on dual-upper-state design, Appl. Phys. Lett. 96 (2010) 241107.

16. K. Fujita, M. Hitaka, A. Ito, T. Edamura, M. Yamanishi, S. Jung and M.A. Belkin, Terahertz generation in midinfrared quantum cascade lasers with a dualupper-state active region, Appl. Phys. Lett. 106 (2015) 251104.

17. M. Razeghi, Q.Y. Lu, N. Bandyopadhyay, W. Zhou, D. Heydari, Y. Bai and S. Slivken, Quantum cascade lasers: from tool to product, Opt. Express. 75 (23) (2015) 8462.

18. K. Vijayraghavan, R. W. Adams, A. Vizbaras, M. Jang, C. Grasse, G. Boehm, M.C. Amann and M. A. Belkin, Terahertz sources based on Čerenkov differencefrequency generation in quantum cascade lasers, Appl. Phys. Lett. 100 (2012)251104.

19. R. L. Sutherland, Handbook of Nonlinear Optics 2nd Ed. (Marcel Dekker INC, New York, 2003) chp. 2, pp. 33-120.

20. J. Faist, D. Hofstetter, M. Beck, T. Aellen, M. Rochat and S. Blaser, Bound-tocontinuum and two-phonon resonance, quantum cascade lasers for high duty cycle, high-temperature operation, IEEE J. Quantum Electron. 38(6) (2002) 533.

21. S. Jung, A. Jiang, Y. Jiang, K. Vijayraghavan, X. Wang, M. Troccoli and M. A. Belkin, Broadly tunable monolithic room-temperature terahertz quantum cascade laser sources, Nat. Commun. 5(2014)4267.

22. Q. Lu and M. Razeghi, Recent Advances in Room temperature, high-power terahertz quantum cascade laser sources based on difference-frequency generation, Photonics 3 (42)(2016)1-10; doi:10.3390/photonics3030042. 
23. K. Fujita, S. Junga, Y. Jiang, J.-H. Kim, A. Nakanishi, A. Ito, M. Hitaka, T. Edamura and M.- A. Belkin, Recent progress in terahertz difference-frequency quantum cascade laser sources, Nanophotonics; aop (2018)1.

24. M. Razeghi, Q. Y. Lu, N. Bandyopadhyay, Y. Bai and S. Slivken, Proc. SPIE 9199, (2014) 919902.

25. N. Hodgson and H. Weber, Laser Resonators and Beam Propagation: Fundamentals, Advanced Concepts and Applications 2nd Ed.(Springer, USA, 2005) chp. 9, pp. 367-392.

26. A. Hamadou, S. Lamari and J.-L. Thobel, Rate equations analysis of a dualwavelength quantum cascade laser, Opt.commu. 305 (2013) 147.

27. A. Hamadou, S. Lamari and J.-L. Thobel, Delay time calculation for dualwavelength quantum cascade lasers, J. Appl. Phys. 114 (2013) 203102.

28. A. Hamadou, J.-L. Thobel and S. Lamari, Dynamic modeling of a terahertz quantum cascade laser based on difference frequency generation, Optik. 156(2018)596.

29. M. Yamanishi, T. Edamura, K. Fujita, N. Akikusa and H. Kan, Theory of the intrinsic linewidth of quantum-cascade lasers: Hidden reason for the narrow linewidth and line-broadening by thermal photons, IEEE J. Quantum Electron. 44 (2008)12.

30. M. Geiser, C. Pflügl, A. Belyanin, Q.-J. Wang, N. Yu, T. Edamura, M. Yamanishi, H. Kan, M. Fischer, A. Wittmann, J. Faist and F. Capasso, Gain competition in dual wavelength quantum cascade lasers, Opt. Express. 18(2010) 9900.

31. Y.-R. Shen, Nonlinear Infrared Generation 4th Ed. (Springer-Verlag Berlin Heidelberg, New York, 1977).

32. S. Jung, J.-H. Kim, Y. Jiang, K. Vijayraghavan and M.-A. Belkin, Terahertz difference-frequency quantum cascade laser sources on silicon, Optica. 4 (2017)38.

33. R.W. Boyd, Nonlinear Optics, 2nd Ed.( Academic Press, San Diego, 2003). 


\section{Figures captions}

Fig.1. Schematic illustration of the Čerenkov THz DFG-QC laser. The nonlinear active region is shown in green and the substrate is in black. Red solid arrows indicate the Čerenkov radiation emission at an angle $\theta_{\mathrm{C}}$. The front facet of the substrate is polished to facilitate the out coupling of the $\mathrm{THz}$ radiation to free space $\left(R_{s u b}^{(T H z)}\right) . R_{1}^{(i)}$ and $R_{2}^{(i)}$ are the intensity reflection coefficients at facets 1 and 2 respectively, for wavelength $\lambda_{i}$, with $i=1,2$.

Fig. 2. Four-level model of one stage of the bound-to-continuum active region in a dual-wavelength QC laser as used in our work. Lasing takes place simultaneously through transitions from 4 onto 3 on one hand and from 4 onto 2 on the other. The $\mathrm{THz}$ frequency is generated via DFG process between levels 3 and 2.

Fig.3. Dependence of the relative efficiency $\ell$ on $\mathrm{THz}$ absorption coefficient in the substrate without and with mid-IR losses. $\alpha_{w}^{(1)}=\alpha_{w}^{(2)}=0 \mathrm{~cm}^{-1}$ (blue solid line), $\alpha_{w}^{(1)}=\alpha_{w}^{(2)}=8 \mathrm{~cm}^{-1}$ (red dashed line).

Fig.4. Dependence of the THz-optical external efficiency on current injection for different cavity lengths and at different values of the THz loss coefficients in the substrate: $\alpha_{\text {sub }}^{(T H z)}=20 \mathrm{~cm}^{-1}$ (blue sold line), $\alpha_{\text {sub }}^{(T H z)}=10 \mathrm{~cm}^{-1}$ (red dashed line), and $\alpha_{\text {sub }}^{(T H z)}=5 \mathrm{~cm}^{-1}$ (green dotted line). The intensity reflection coefficient of the substrate $R_{\text {sub }}^{(T H z)}=30 \%$. 
Fig.5. Calculated contour plots of the THz-optical external efficiency $\eta_{\text {ext }}^{(T H z)}(\%)$ as a function of the cavity length and $\mathrm{THz}$ loss in the substrate. The injected current $I=10 \mathrm{~A}$, and the intensity reflection coefficient of the substrate $R_{s u b}^{(T H z)}=30 \%$.

Fig. 6. Dependence of the mid-IR and THz DFG-QC laser optical external efficiencies on the mid-IR reflecting power $R_{1}$. Blue solid line and red dashed one are for mode pumps lasing at $10.5 \mu \mathrm{m}$ and $8.9 \mu \mathrm{m}$, while black dotted line is for $\mathrm{THz}$ DFG. The injected current $I=10 \mathrm{~A}$, the losses in the substrate $\alpha_{\text {sub }}^{(T H z)}=5 \mathrm{~cm}^{-1}$, the nonlinear cavity length $L=1 \mathrm{~mm}$, and the intensity reflection coefficient of the substrate $R_{s u b}^{(T H z)}=30 \%$.

Fig.7. Dependence of the THz-optical external efficiency on the THz losses in the substrate for $R_{1}=0.29$ (blue solid line) and $R_{1}=1$ (red dashed line). The intensity reflection coefficient of the substrate $R_{\text {sub }}^{(T H z)}=30 \%$, the injected current $I=10 \mathrm{~A}$, and the nonlinear cavity length $L=1 \mathrm{~mm}$. 


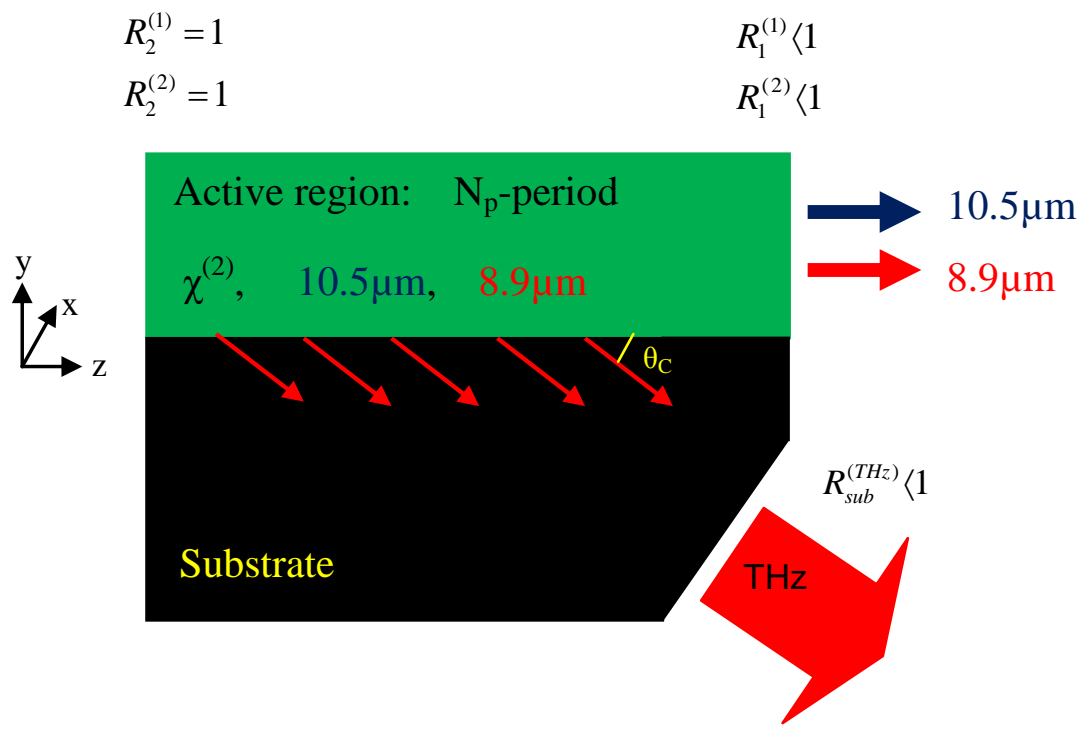

Title: $\quad$ Optical external efficiency of terahertz quantum cascade laser based on Čerenkov difference frequency generation

Authors: A. Hamadou, J.-L. Thobel and S. Lamari

FIG. 1 


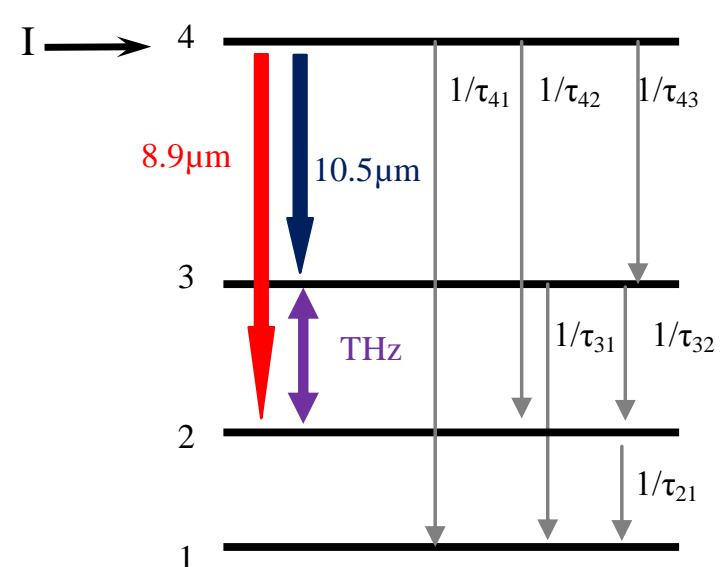

Title: Optical external efficiency of terahertz quantum cascade laser based on Čerenkov difference frequency generation

Authors: A. Hamadou, J.-L. Thobel and S. Lamari

FIG. 2 


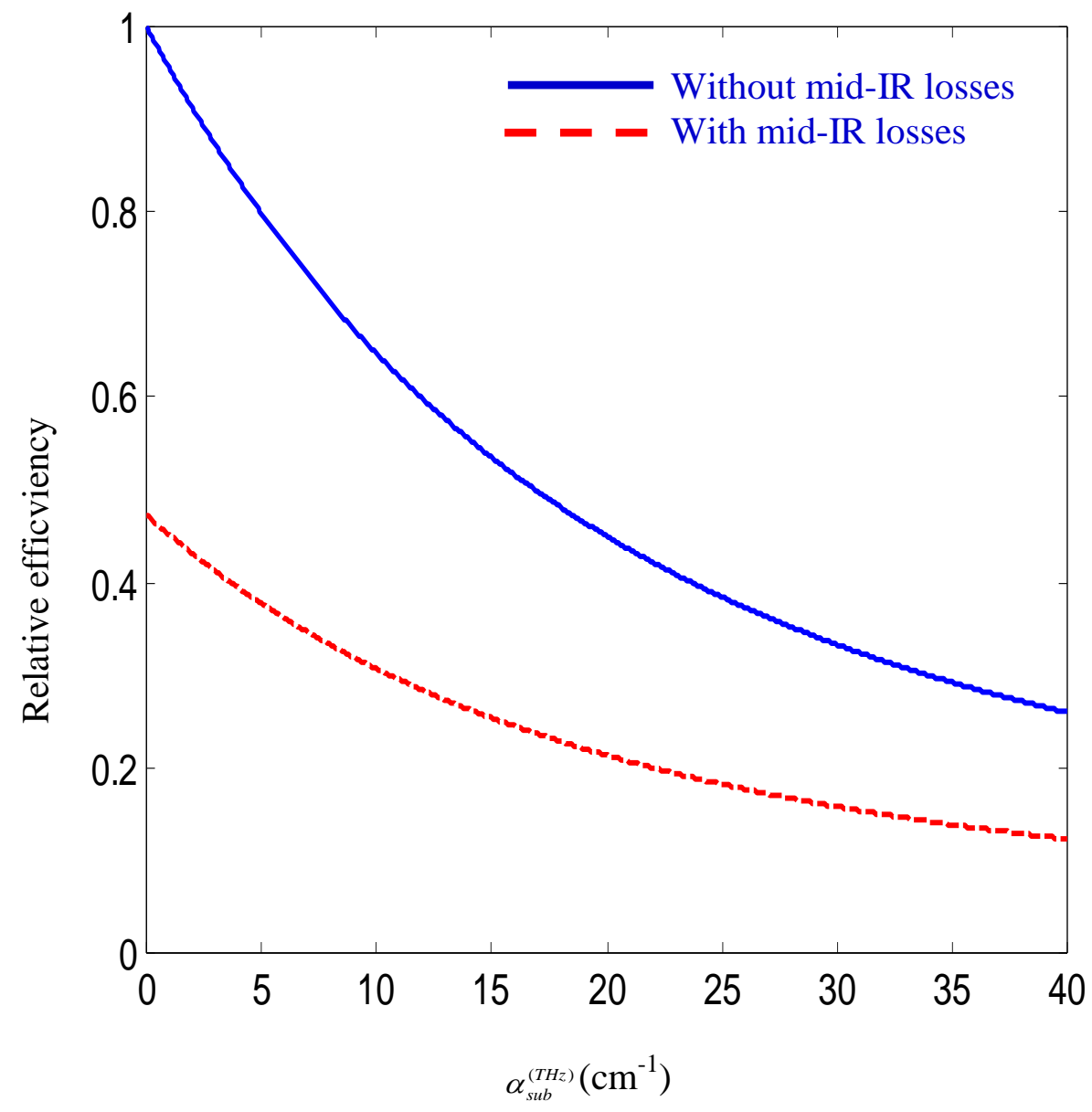

Title: $\quad$ Optical external efficiency of terahertz quantum cascade laser based on Čerenkov difference frequency generation

Authors: A. Hamadou, J.-L. Thobel and S. Lamari

FIG. 3 

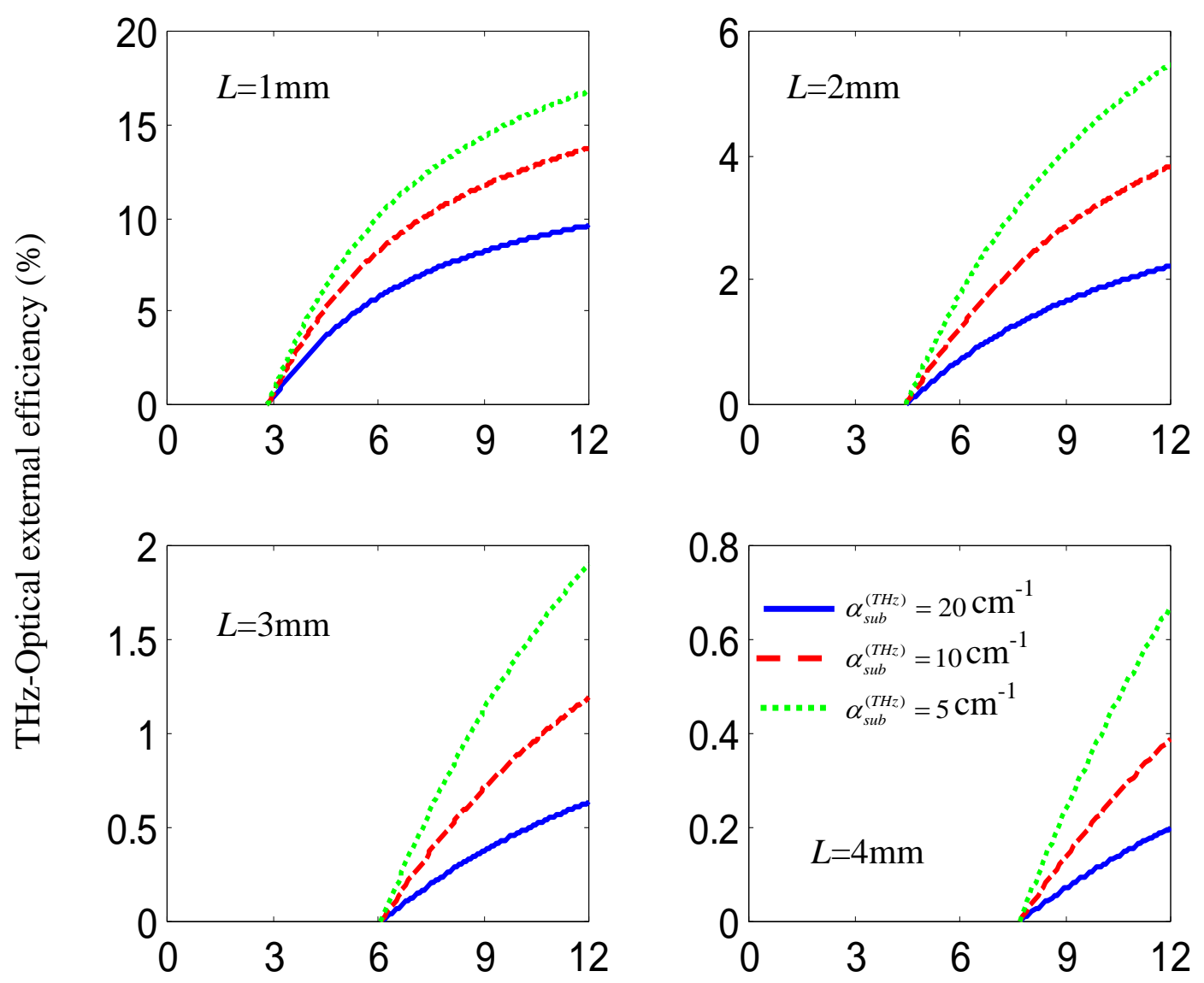

Current (A)

Title: $\quad$ Optical external efficiency of terahertz quantum cascade laser based on Čerenkov difference frequency generation

Authors: A. Hamadou, J.-L. Thobel and S. Lamari

FIG. 4 


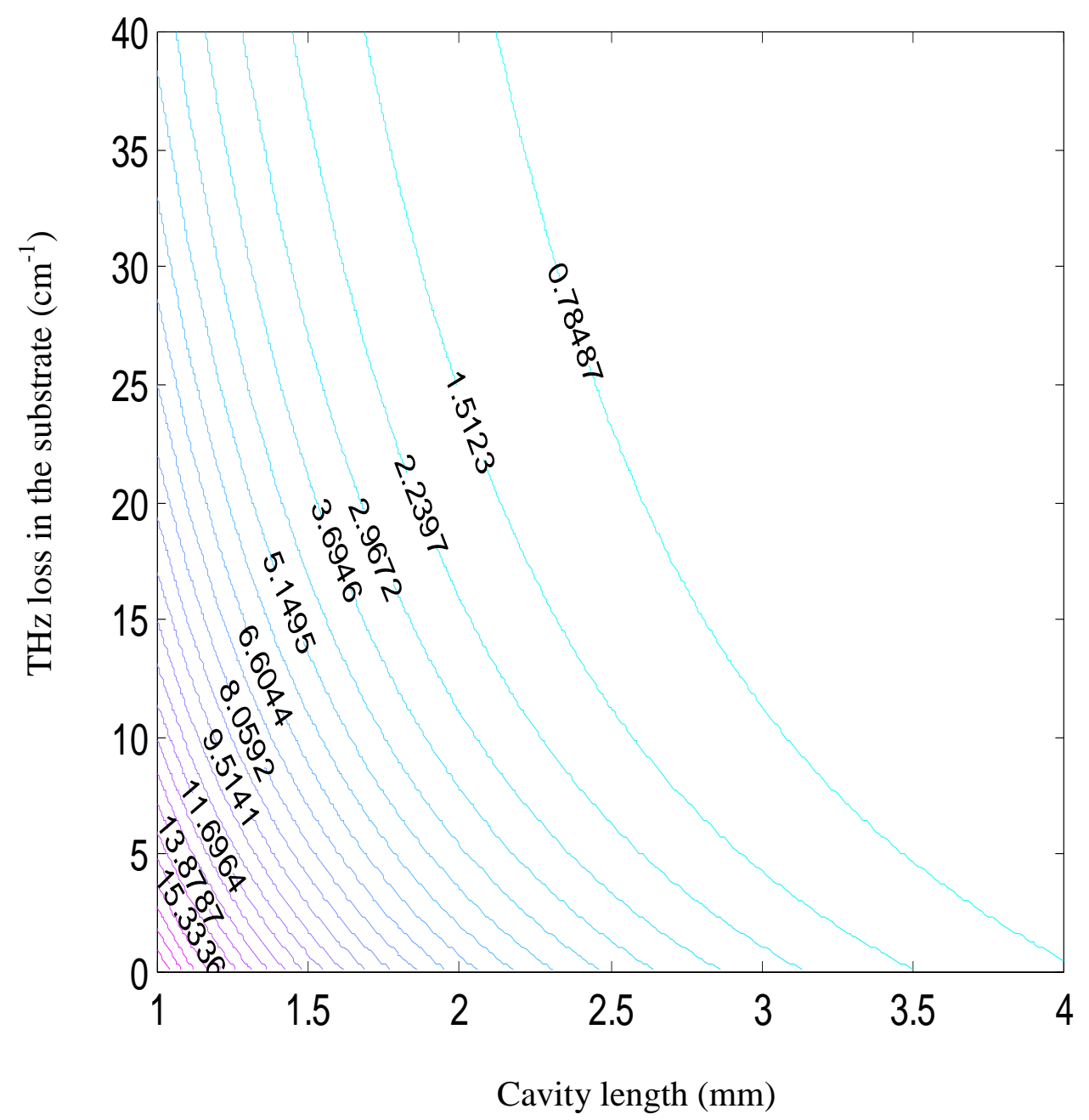

Title: Optical external efficiency of terahertz quantum cascade laser based on Čerenkov difference frequency generation

Authors: A. Hamadou, J.-L. Thobel and S. Lamari

FIG. 5 


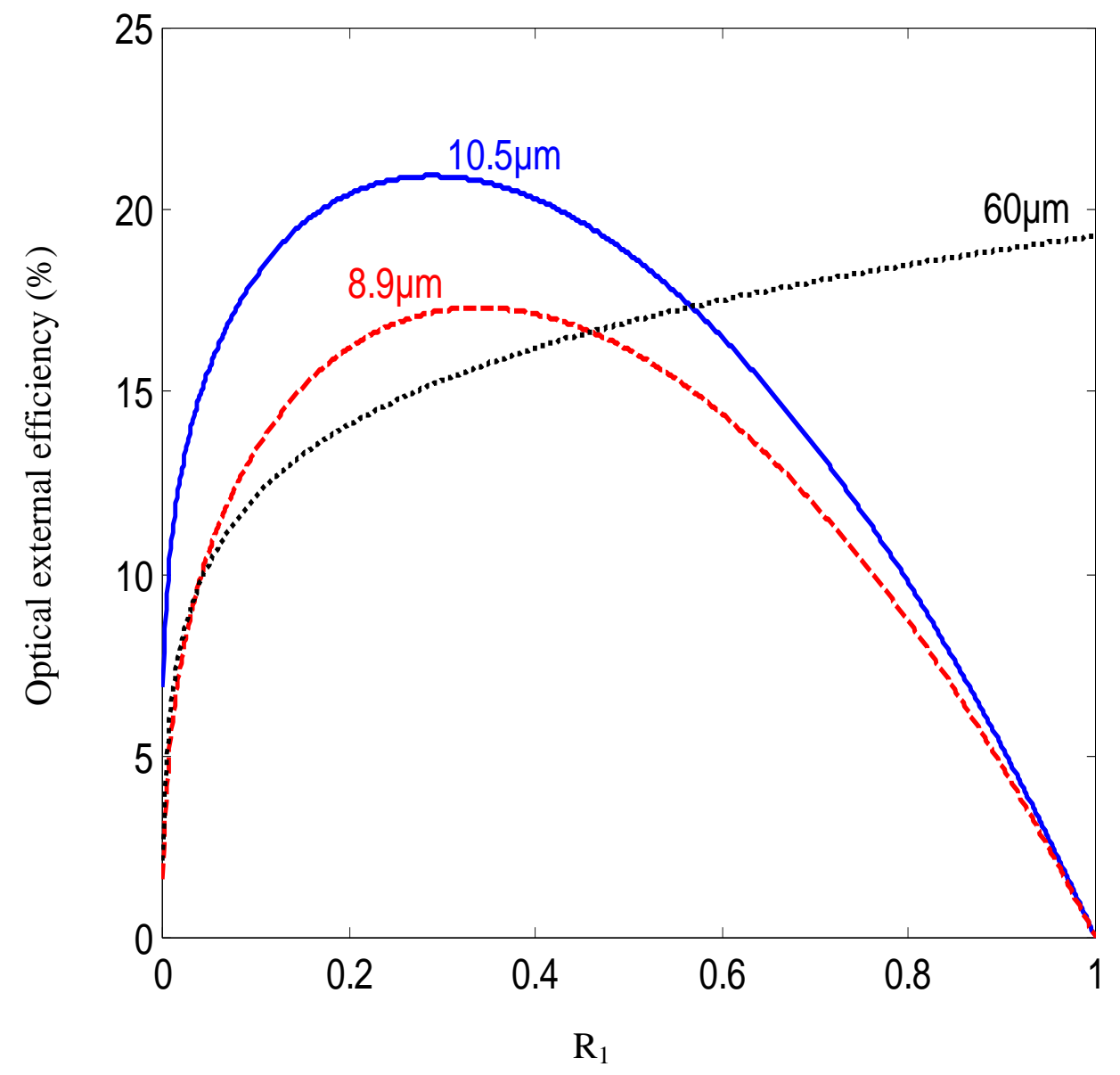

Title: $\quad$ Optical external efficiency of terahertz quantum cascade laser based on Čerenkov difference frequency generation

Authors: A. Hamadou, J.-L. Thobel and S. Lamari

FIG. 6 


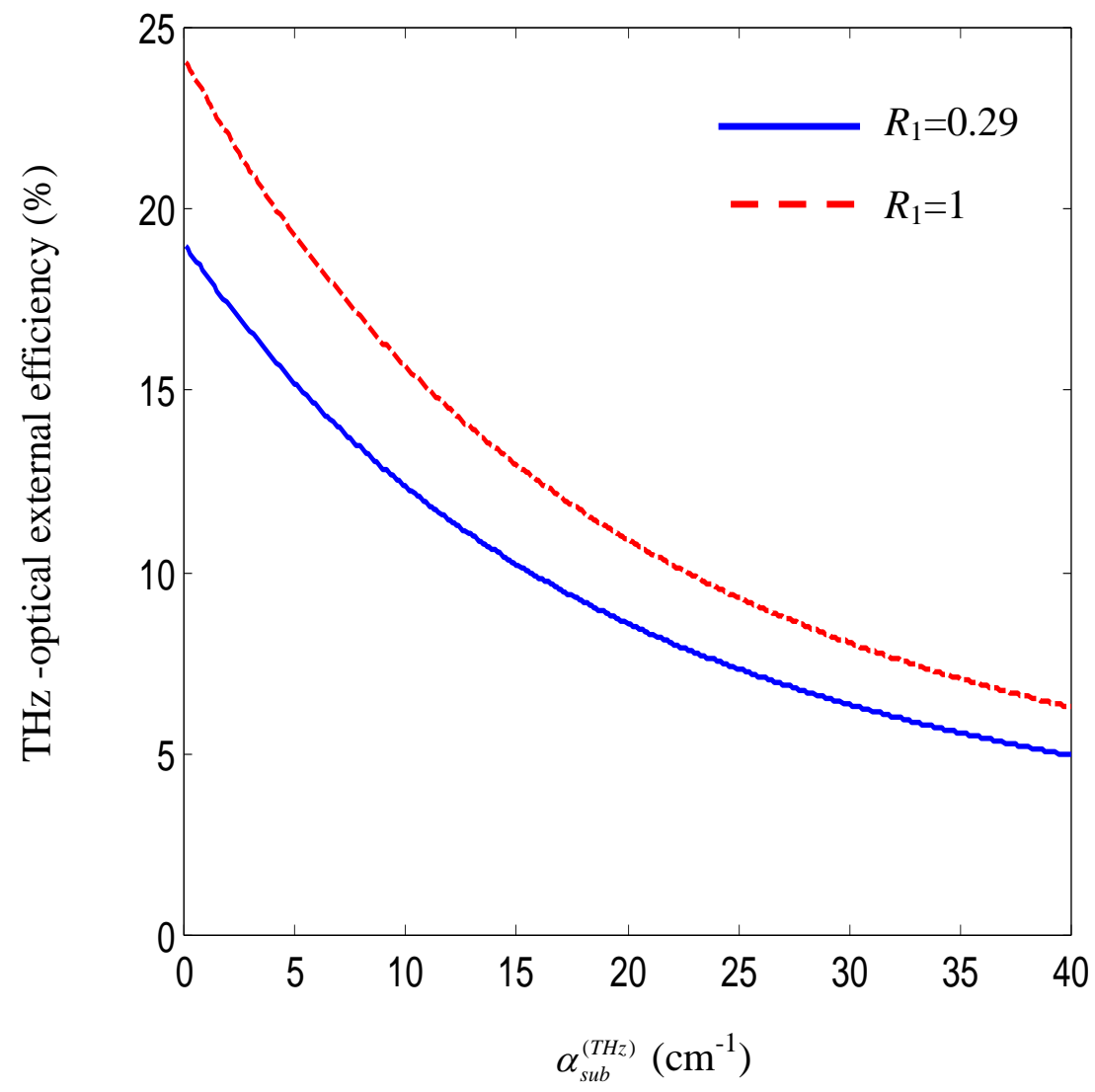

Title: $\quad$ Optical external efficiency of terahertz quantum cascade laser based on Čerenkov difference frequency generation

Authors: A. Hamadou, J.-L. Thobel and S. Lamari

FIG. 7 